\title{
Efficacy and effectiveness trials have different goals, use different tools, and generate different messages [Corrigendum]
}

\author{
Porzsolt F, Rocha NG, Toledo-Arruda AC, et al. Pragmatic \\ and Observational Research. 2015;6:47-54.
}

The authors wish to advise that the Introduction section is missing this final sentence: on page 48, left column, line 34, "A short movie provides additional information on this topic https://drive. google.com/file/d/0B9MPdcimmmmpQkQ0cjdKNDk0R0U/ view". 